\title{
The black hole mass of BL Lacs from stellar velocity dispersion of the host galaxy.
}

\author{
Renato Falomo ${ }^{1}$, Jari Kotilainen ${ }^{2}$, and Aldo Treves ${ }^{3}$ \\ 1 Osservatorio Astronomico di Padova, Vicolo dell'Osservatorio 5, Padova, Italy \\ 2 Tuorla Observatory, University of Turku, Väisäläntie 20, Piikkiö, Finland \\ 3 Università dell'Insubria, Via Valleggio 11, Como, Italy
}

\section{Introduction}

One of the most important quantities in theoretical models of AGNs is the black hole mass $\left(\mathrm{M}_{B H}\right)$ that, together with the total luminosity, defines the fraction of the Eddington luminosity at which the AGN is emitting. Determination of $\mathrm{M}_{B H}$ in AGN is difficult mainly because of the bright emission from the nucleus and their large distance. The main method that has proved to be successful in AGN is reverberation mapping, which is extremely time consuming and gives results on $\mathrm{M}_{B H}$ that depend on the assumed geometry of the accretion disk. Therefore, only for a few well studied quasars and Seyfert galaxies $\mathrm{M}_{B H}$ is known (see e.g. [7], [11] and references therein). This method cannot obviously be employed for BL Lac objects because they lack prominent emission lines. Therefore other methods need to be applied to infer $\mathrm{M}_{B H}$ for BL Lacs. The discovery of a relation between $\mathrm{M}_{B H}$ and the luminosity of the bulge in nearby early-type galaxies offers now a new tool for estimating the mass of the central BH (see e.g. review [10]). This has been done for two samples of nearby quasars [9] and BL Lacs [12].

Recently, a tighter correlation was found relating $\mathrm{M}_{B H}$ with the central stellar velocity dispersion $\sigma$ of the spheroidal component in nearby galaxies [5,2], that can also be used to estimate $\mathrm{M}_{B H}$ in AGN. The relationship appears to predict more accurately [10] $\mathrm{M}_{B H}$, but requires the measurement of $\sigma$ in the host galaxies of AGN that is difficult to obtain, in particular for objects at moderately high redshift and with very luminous nuclei. On the other hand, for BL Lacs that have relatively fainter nuclei than quasars, this measurement (at least for low redshift objects) can be secured with observations at medium-sized telescopes.

We present here the first estimates of stellar velocity dispersion of BL Lacs from our ongoing program aimed specifically at deriving $\mathrm{M}_{B H}$ from the $\mathrm{M}_{B H}-$ $\sigma$ correlation. We selected a sample of nearby $(\mathrm{z}<0.2)$ BL Lacs for which high quality images were obtained either from the ground using the Nordic Optical Telescope (NOT) [3] or with HST+WFPC2 [13,4]. From the images a characterization of the host galaxies and of the nuclear luminosity are obtained. This allows us to compare $\mathrm{M}_{B H}$ with the mass (and the luminosity) of the host galaxy and also to evaluate the Eddington ratio, provided that the nuclear emitted power is corrected for the beaming factor. Moreover, a comparison of $\mathrm{M}_{B H}$ for BL Lacs with different jet/ disk luminosities can be used to test the hypothesis (see e.g. [8]) that the accretion rate changes from largely sub-Eddington, for low lumi- 
nosity weak-lined sources, to near-Eddington for high luminosity, strong-lined sources. If the accretion rate in terms of Eddington ratio were the same in both classes, the $\mathrm{BH}$ masses should differ almost by three orders of magnitude.

\section{Observations, data analysis and first results}

We secured medium resolution ( $\mathrm{R} \sim 3000$ ) optical spectra of a sample of the BL Lacs using the NOT $2.5 \mathrm{~m}$ and the ESO $3.6 \mathrm{~m}$ telescopes equipped with ALFOSC and EFOSC2, respectively. The chosen grisms combined with a 1 arcsec slit yield a spectral resolution for velocity dispersion measurement of $\sim 60-80 \mathrm{~km} / \mathrm{s}$. The used spectral range includes the absorption lines of Ca II (3933-68 $\AA), \mathrm{Mg}$ I $(5175 \AA)$, E-band $(5269 \AA)$ and Na I $(5892 \AA)$ from the host galaxies. The measurement of $\sigma$ was done with the Fourier Quotient method using template spectra of late-type ( $\mathrm{G}$ and $\mathrm{K})$ stars.

Here we report the first results on three BL Lacs: Mkn 501, Mkn 180 and PKS 2201+04. From the measurement of $\sigma$ we have evaluated the black hole masses assuming that the $\mathrm{M}_{B H}-\sigma$ relationship [2] is valid for the BL Lac hosts. We found similar BH masses $\left(\log \left(\mathrm{M}_{B H}\right)=8.5\right.$ and 8.7) for Mkn 501 and Mkn 180 , and a significantly lower value $\left(\log \left(\mathrm{M}_{B H}\right)=7.5\right)$ for PKS $2201+04$.

From the values of $\sigma$ and the effective radius $\mathrm{R}_{e}$ we have also estimated the mass of the host galaxy (using the relation $\mathrm{M}($ host $)=5 \mathrm{R}_{e} \sigma^{2} / \mathrm{G}$; [1] and compared it with that of the $\mathrm{BH}$. It turns out that $\mathrm{M}_{B H} / \mathrm{M}$ (host) for the $\mathrm{BL}$ Lacs is in the range of $0.03-0.1 \%$.

\section{References}

1. Bender, R., Burstein,D., Faber, S.M. 1992, ApJ 399, 462

2. Ferrarese L., Merritt D, 2000, ApJ 539, L9

3. Falomo, R., Kotilainen, J., 1999, A\&A 352, 85

4. Falomo et al 2001, ApJ 547, 124

5. Gebhardt et al. 2000, ApJ 539, L13

6. Haehnelt M.G. and Kauffmann G.,2000, MNRAS 318, L35

7. Kaspi et al 2000, ApJ 533, 631

8. Maraschi, L. \& Tavecchio F., 2000, (astro-ph/0102295)

9. McLure \& Dunlop 2001 (astro-ph/0108417)

10. Merritt D \& Ferrarese L. , 2001, astro-ph/0107134

11. Nelson C.H., 2000, ApJ 544, L91

12. Treves, A., et al, ASP Conf Series, in press (astro-ph/0107129

13. Urry C.M., et al, 2000, ApJ 532, 816 